06

\title{
Феноменологическая модель нелинейных оптических свойств топологической среды
}

\author{
(C) А.И. Маймистов \\ Национальный исследовательский ядерный университет МИФИ, \\ 115409 Москва, Россия \\ e-mail: aimaimistov@gmail.com
}

Поступила в редакцию 11.09.2020 г.

В окончательной редакции 11.09.2020 г.

Принята к публикации 22.09.2020 г.

Исходя из квазиклассического описания динамика электронов в топологических средах, гиротропные свойства которых обусловлены ненулевой кривизной Берри, предложена феноменологическая модель, которая позволяет рассмотреть некоторые нелинейные оптические процессы - генерацию третьей гармоники и поворот вектора поляризации волны, зависящий от интенсивности. Определены нелинейные восприимчивости третьего порядка, которые определяют взаимодействие волн с учетом вращения вектора поляризации волн. Гиротропия проявляется в зависимости эффективности преобразования в гармонику от циркулярной поляризации излучения, что может быть использовано для измерений параметров таких сред.

Ключевые слова: связность Берри, кривизна Берри, топологический изолятор, нелинейная поляризуемость, гиротропная среда, генерация третьей гармоники.

DOI: $10.21883 /$ OS.2021.01.50443.231-20

\section{Введение}

В физике есть ряд примеров использования феноменологических теорий, для которых детальная природа явлений либо не известна, либо известна только в общих чертах. Но при этом достигается качественное описание явлений. Например, представление поляризации диэлектрика рядом по степеням напряженности электрического поля позволяет развить теорию нелинейных оптических явлений, таких как генерация гармоник, параметрическое взаимодействие полей, самофокусировка и автомодуляция, нелинейное поглощений и комбинационное рассеяние [1-3], распространение оптических солитонов [4]. При этом коэффициенты разложения поляризации, называемые тензорами нелинейной восприимчивости соответствующих порядков, не вычисляются, но считаются заданными. Полагая поляризацию зависящей не только от напряженности электрического поля, но и от напряженности магнитного поля, таким же путем можно получить теорию магнитоэлектрических явлений. В общем случае используется разложение термодинамического потенциала (например, свободной энергии) по степеням полей или соответствующего параметра порядка как в теории Ландау фазовых переходов второго рода [5], в теории сверхпроводимости Гинзбург-Ландау [6-8] и в теории сверхтекучести (бозе-конденсата) Гросса-Питаевского [9,10]. Перечень примеров можно было бы продолжить. Некоторые феноменологические модели позже обосновывались микроскопическими моделями, но и без того они играют важную роль в физике.
Всякая феноменологическая модель опирается на некоторые предположения. В настоящей работе будет предложено феноменологическое описание нелинейных оптических процессов в топологических средах, рассматривая движение электронов в кристалле. В квазиклассическом приближении динамика электронов (т.е. заряженных квазичастиц в кристалле) описывается как движение волнового пакета под действием электрического и магнитного полей [11]. Положение центра волнового пакета и сопряженный импульс удовлетворяют уравнениям Гамильтона

$$
\dot{\mathbf{r}}=\frac{\partial E(\mathbf{p})}{\partial \mathbf{p}}=\mathbf{v}(\mathbf{p}), \quad \dot{\mathbf{p}}=e \mathbf{E}+\frac{e}{c} \dot{\mathbf{r}} \times \mathbf{B},
$$

где $\mathbf{E}$ и В есть напряженности внешнего электрического и магнитного полей, $E(\mathbf{p})-$ кинетическая энергия электрона в разрешенной зоне, $\mathbf{r}$ - положение центра волнового пакета зонных электронов, р - вектор квазиимпульса ${ }^{1}$, сопряженного координате $\mathbf{r}, c-$ скорость света и точка над векторами означает взятие производной по времени. При выводе уравнений (1) электроны рассматривались как свободные частицы. Известно, что состояние электрона в периодическом поле кристаллической решетки описывается векторами состояния Блоха $|\psi\rangle=\exp (i \mathbf{p r} / \hbar)\left|u_{\mathbf{p}}\right\rangle$, где $\mathbf{p}=\hbar \mathbf{k}-$ квазиимпульс электрона, $\left|u_{\mathbf{p}}(\mathbf{r})\right\rangle=\left|u_{\mathbf{p}}\left(\mathbf{r}+\mathbf{r}_{\text {lat }}\right)\right\rangle$ для любого вектора прямой решетки $\mathbf{r}_{\text {lat }}$. Состояния Блоха дополнительно индексируются номерами зон. Предположим, что межзонных переходов нет, тогда номер зоны можно опустить. С учетом блоховского характера вектора состояния электрона

\footnotetext{
${ }^{1}$ Обычно используется вектор квазиимпульса $\mathbf{k}$, вместо которого здесь берется $\mathbf{p}=\hbar \mathbf{k}$.
} 
были уточнены уравнения движения волнового пакета и получена следующая система уравнений $[12,13]$ :

$$
\dot{\mathbf{r}}=\mathbf{v}(\mathbf{p})+\dot{\mathbf{p}} \times \mathbf{\Omega}(\mathbf{p}), \quad \dot{\mathbf{p}}=e \mathbf{E}+\frac{e}{c} \dot{\mathbf{r}} \times \mathbf{B},
$$

в которых учитывается аномальная скорость. Эта поправка к скорости содержит вектор кривизны Берри $\boldsymbol{\Omega}$, компоненты которого выражаются через вектор $\mathbf{A}(\mathbf{p})$, называемый связностью Берри, соотношениями $\Omega_{i}=\varepsilon_{i j k} \partial A_{k} / \partial p_{j}$. (Здесь используется единичный антисимметричный псевдотензор третьего ранга, имеющий компоненты $\varepsilon_{i j k}$, чтобы представить вектор $\Omega$ как ротор в импульсном пространстве вектора $\mathbf{A}(\mathbf{p})$.) Связность Берри определяется выражением $\mathbf{A}(\mathbf{p})=i\left\langle u_{\mathbf{p}}|\partial / \partial \mathbf{p}| u_{\mathbf{p}}\right\rangle$. Таким образом, нетривиальные свойства зон Бриллюэна могут приводить к ненулевому значению кривизны Берри $\boldsymbol{\Omega}(\mathbf{p})$, с помощью которой определяются топологические свойства среды [12-14].

В известной модели ангармонического осциллятора (модели Лоренца) нелинейный отклик среды является результатом негармонического движения заряженной частицы во внешнем электромагнитном поле $[1,2]$. В рассматриваемой модели ангармонического потенциала нет, но негармоническое движение волнового пакета обусловлено сложной (не линейной) зависимостью скорости $\mathbf{v}(\mathbf{p})$, зависимостью кривизны Берри $\boldsymbol{\Omega}(\mathbf{p})$ от квазиимпульса и силой Лоренца (второе слагаемое в уравнении для импульса).

В настоящей работе сила Лоренца, которая содержит малый параметр $c^{-1}$, не будет учитываться, тем самым магнитоэлектрические явления модель не отражает. Нелинейный отклик среды, обусловленный только силой Лоренца, был рассмотрен в [15]. Была рассмотрена обобщенная модель Друде-Лоренца, учитывающая топологические эффекты в минимальной степени, т.е. в предположении, что $E(\mathbf{p})=\mathbf{p}^{2} / 2 m$ и $\boldsymbol{\Omega}$ является постоянным вектором. В этом приближении описаны известные закономерности линейной электродинамики топологических сред и были получены выражения нелинейной проводимости второго порядка, отвечающей за генерацию второй гармоники и эффект оптического выпрямления. Естественным обобщением был бы учет непараболической зависимости энергии электронов от импульса и зависимости вектора кривизны Берри от импульса. Здесь будут рассмотрена упрощенная модель, в которой эффекты, связанные с магнитным полем (силой Лоренца) не учитываются, но нелинейный отклик среды обусловлен более сложными зависимостями от импульса величин $E(\mathbf{p})$ и $\boldsymbol{\Omega}(\mathbf{p})$. Известно, что полуметаллу Вейля присуща гиротропия в отсутствии магнитного поля [16,17]. Интересно выяснить, каким образом это характерно топологическое свойство проявится в классических нелинейных явлениях, таких как параметрическое взаимодействие и самовоздействие.

\section{1. Поляризация среды}

Движение электронов в квазиклассическом приближении описывается системой уравнений

$$
\dot{\mathbf{r}}=\mathbf{v}(\mathbf{p})+\dot{\mathbf{p}} \times \Omega(\mathbf{p}), \quad \dot{\mathbf{p}}+\gamma \mathbf{p}=e \mathbf{E},
$$

где $m$ - эффективная масса, и слагаемое с коэффициентом $\gamma$ обеспечивает релаксацию импульса. Зависимость вектора $\boldsymbol{\Omega}(\mathbf{p})$ от импульса приведет к тому, что поляризация среды, определенная формулой $\mathbf{P}(t)=n_{e} e \mathbf{r}(t)$, будет нелинейно зависеть от напряженности электрического поля $\mathbf{E}$. Прежде чем рассмотреть нелинейные свойства среды полезно рассмотреть случай, когда $\mathbf{v}(\mathbf{p})=\mathbf{p} / m$ и $\boldsymbol{\Omega}(\mathbf{p})=\Omega_{0} \mathbf{n}$ при $\mathbf{n}-$ постоянном векторе единичной длины.

\section{1. Линейное приближение}

Если $\boldsymbol{\Omega}(\mathbf{p})=\Omega_{0} \mathbf{n}$, то преобразование Фурье превратит систему уравнений (3) в алгебраические уравнения, из решения которых следует выражение

$$
\mathbf{r}(\omega)=\frac{(-1) e}{m \omega(\omega+i \gamma)} \mathbf{E}(\omega)-\frac{i \Omega_{0} e}{\omega+i \gamma} \mathbf{n} \times \mathbf{E}(\omega) .
$$

Для фурье-компоненты поляризации $\mathbf{P}(\omega)=n_{e} e \mathbf{r}(\omega)$ (здесь $n_{e}$ - концентрация квазичастиц, имеющих заряд $e$ и эффективную массу $m$ ) отсюда следует, что

$$
\mathbf{P}(\omega)=-\frac{e^{2} n_{e}}{m \omega(\omega+i \gamma)} \mathbf{E}(\omega)-\frac{i \Omega_{0} e^{2} n_{e}}{\omega+i \gamma} \mathbf{n} \times \mathbf{E}(\omega) .
$$

Таким образом, выражение для электрической индукции имеет вид

$$
\mathbf{D}(\omega)=\left(1-\frac{\omega_{p}^{2}}{\omega(\omega+i \gamma)}\right) \mathbf{E}(\omega)-\frac{i \Omega_{0} 4 \pi e^{2} n_{e}}{\omega+i \gamma} \mathbf{n} \times \mathbf{E}(\omega) .
$$

В (5) использовано выражение для квадрата плазменной частоты $\omega_{p}^{2}=4 \pi e^{2} n_{e} / m$. Первое слагаемое в (5) есть линейная диэлектрическая проницаемость, обусловленная электронами в незаполненной зоне. Вклад от остальных электронов приведет к тому, что выражение в круглых скобках надо будет заменить линейной диэлектрической проницаемостью среды $\varepsilon(\omega)$. Второе слагаемое в (5) обусловлено топологическими свойствами среды и описывает вращение поляризации - среда является гиротропной. Для вектора гирации получено отсюда выражение

$$
\mathbf{g}(\omega)=\frac{\Omega_{0} m \omega_{p}^{2}}{(\omega+i \gamma)} \mathbf{n}
$$

В работах [17-19], где рассматривались электромагнитные свойства полуметалла Вейля [20,21], представлено выражение для электрической индукции этого материала:

$$
\mathbf{D}(\omega)=\varepsilon(\omega) \mathbf{E}(\omega)-\frac{i e^{2}}{\pi \hbar \omega} 2 \mathbf{b} \times \mathbf{E}(\omega)-\frac{i e^{2}}{\pi \hbar \omega c} 2 b_{0} \mathbf{B}(\omega),
$$


где $2 \mathbf{b}$ - вектор в импульсном пространстве, соединяющий два узла Вейля, и $2 \hbar b_{0}$ - расстояние между этими узлами на оси энергии. Сравнивая (5) и (6), можно выразить параметр рассматриваемой модели через характеристики полуметалла Вейля: $\Omega_{0} n_{e}=2 b / 4 \pi^{2} \hbar$ и $b_{0}=0$.

\section{2. Нелинейная поляризация}

Поправки к поляризации можно получить, опираясь на следующие предположения: (а) $E(\mathbf{p})=\mathbf{p}^{2} / 2 m+$ $+\kappa \mathbf{p}^{4} / 4$, так что $\mathbf{v}(\mathbf{p})=\mathbf{p} / m+\kappa(\mathbf{p} \cdot \mathbf{p}) \mathbf{p}$, и (б) $\boldsymbol{\Omega}(P)=$ $=\left(\Omega_{0}+\Omega_{2} \mathbf{p}^{2}\right) \mathbf{n}$.

В предположении, что электромагнитное поле монохроматическое, т.е. когда $\mathbf{E}=\mathbf{E}_{0} e^{-i \omega t}+\mathbf{E}_{0}^{*} e^{i \omega t}$, второе уравнение системы (3) даст выражение для поляризации $\mathbf{p}=\mathbf{p}_{0} e^{-i \omega t}+\mathbf{p}_{0}^{*} e^{i \omega t}$, где

$$
\mathbf{p}_{0}=\frac{i e}{(\omega+i \gamma)} \mathbf{E}_{0}
$$

Подстановка этого выражения в первое уравнение системы (3) приводит к выражению, которое содержит осциллирующие слагаемые на основной частоте $\omega$ и на частоте $3 \omega$. Следовательно, координата $\mathbf{r}$ должна быть представлена как

$$
\mathbf{r}=\mathbf{r}_{0} e^{-i \omega t}+\mathbf{r}_{0}^{*} e^{i \omega t}+\mathbf{r}_{3} e^{-i 3 \omega t}+\mathbf{r}_{3}^{*} e^{i 3 \omega t} .
$$

Это ситуация типичная для ангармонической модели Лоренца (модели Дюффинга) [1-3]. Слагаемые, отвечающие колебаниям на нулевой частоте (оптическое выпрямление) и на удвоенной частоте (генерации второй гармоники), появились бы в случае кубической поправки к энергии квазичастиц (электронов) и линейной поправке по импульсу у кривизны Берри.

Подстановка (7) в первое уравнение системы (3) позволяет найти явно выражения для коэффициентов $\mathbf{r}_{0}=\mathbf{r}_{0 l i n}+\mathbf{r}_{0 \text { nl }}$ и $\mathbf{r}_{3}$ :

$$
\begin{gathered}
\mathbf{r}_{0 l i n}(\omega)=\frac{-e}{m \omega(\omega+i \gamma)} \mathbf{E}(\omega)-\frac{i \Omega_{0} e}{\omega+i \gamma} \mathbf{n} \times \mathbf{E}(\omega), \\
\mathbf{r}_{0 n l}(\omega)=\frac{-\kappa e^{3}}{\omega\left(\omega^{2}+\gamma^{2}\right)(\omega+i \gamma)}\left[2\left(\mathbf{E}_{0} \cdot \mathbf{E}_{0}^{*}\right) \mathbf{E}_{0}+\left(\mathbf{E}_{0} \cdot \mathbf{E}_{0}^{*}\right) \mathbf{E}_{0}\right] \\
+i \frac{\Omega_{2} e^{3}}{\left(\omega^{2}+\gamma^{2}\right)(\omega+i \gamma)}\left[2\left(\mathbf{E}_{0} \cdot \mathbf{E}_{0}^{*}\right) \mathbf{E}_{0}+\left(\mathbf{E}_{0} \cdot \mathbf{E}_{0}^{*}\right) \mathbf{E}_{0}\right] \times \mathbf{n} \\
\mathbf{r}_{3}(\omega)=\frac{\kappa e^{3}}{3 \omega(\omega+i \gamma)^{3}}\left(\mathbf{E}_{0} \cdot \mathbf{E}_{0}\right) \mathbf{E}_{0} \\
+i \frac{\Omega_{2} e^{3}}{3(\omega+i \gamma)^{3}}\left(\mathbf{E}_{0} \cdot \mathbf{E}_{0}\right) \mathbf{n} \times \mathbf{E}_{0} .
\end{gathered}
$$

Используя эти выражения, можно определить поляризации, описывающие различные явления для рассматриваемой модели. Поляризация, отвечающая за линейный отклик среды на гармоническое поле, дается выражением (4). За генерацию третьей гармоники (ГТГ) отвечает поляризация

$$
\begin{aligned}
\mathbf{P}_{\mathrm{THG}}(3 \omega)= & \chi^{(3)}(3 \omega)\left(\mathbf{E}_{0} \cdot \mathbf{E}_{0}\right) \mathbf{E}_{0} \\
& +i \widetilde{\chi}^{(3)}(3 \omega)\left(\mathbf{E}_{0} \cdot \mathbf{E}_{0}\right) \mathbf{n} \times \mathbf{E}_{0},
\end{aligned}
$$

где введены следующие восприимчивости третьего порядка:

$$
\chi^{(3)}(3 \omega)=\frac{\kappa n_{e} e^{4}}{3 \omega(\omega+i \gamma)^{3}}, \quad \tilde{\chi}^{(3)}(\omega)=\frac{\Omega_{2} n_{e} e^{4}}{3(\omega+i \gamma)^{3}} .
$$

Чтобы получить поляризацию, отвечающую за превращения волны третьей гармоники обратно в волну накачки, необходимо положить электромагнитное поле бигармоническим, т. е. $\mathbf{E}=\mathbf{E}_{0} e^{-i \omega t}+\mathbf{E}_{0}^{*} e^{i \omega t}+$ $+\mathbf{E}_{3} e^{-i 3 \omega t}+\mathbf{E}_{3}^{*} e^{i 3 \omega t}$, и повторить все вычисления, найдя из второго уравнения системы (3) $\mathbf{p}=\mathbf{p}_{0} e^{-i \omega t}+$ $+\mathbf{p}_{0}^{*} e^{i \omega t}+\mathbf{p}_{3} e^{-i 3 \omega t}+\mathbf{p}_{3}^{*} e^{i 3 \omega t}$, где

$$
\mathbf{p}_{0}=\frac{i e}{(\omega+i \gamma)} \mathbf{E}_{0}, \quad \mathbf{p}_{3}=\frac{i e}{(3 \omega+i \gamma)} \mathbf{E}_{3} .
$$

Как обычно, появятся новые слагаемые, отвечающие за генерацию следующих гармоник и изменение показателя преломления на частотах гармоник. Здесь эти процессы рассматриваться не будут.

За явления самовоздействия отвечает поляризация $\mathbf{P}_{n l}(\omega)$, где

$$
\begin{aligned}
& \mathbf{P}_{n l}(\omega)=\chi^{(3)}(\omega ; \omega,-\omega, \omega)\left[2\left(\mathbf{E}_{0} \cdot \mathbf{E}_{0}^{*}\right) \mathbf{E}_{0}+\left(\mathbf{E}_{0} \cdot \mathbf{E}_{0}\right) \mathbf{E}_{0}^{*}\right] \\
& -i \chi_{g}^{(3)}(\omega ; \omega,-\omega, \omega) \mathbf{n} \times\left\lfloor 2\left(\mathbf{E}_{0} \cdot \mathbf{E}_{0}^{*}\right) \mathbf{E}_{0}-\left(\mathbf{E}_{0} \cdot \mathbf{E}_{0}\right) \mathbf{E}_{0}^{*}\right\rfloor .
\end{aligned}
$$

Коэффициент в первом слагаемом есть нелинейная восприимчивость третьего порядка

$$
4 \pi \chi^{(3)}(\omega ; \omega,-\omega, \omega)=\frac{(-\kappa) e^{2} m \omega_{p}^{2}}{\omega(\omega+i \gamma)\left(\omega^{2}+\gamma^{2}\right)} .
$$

С ним связаны нелинейное поглощение и процессы самовоздействия (самофокусировка и автомодуляция). Второе слагаемое в (8) является нелинейной поправкой к вектору гирации. Для описания этого явления введена нелинейная восприимчивость третьего порядка

$$
\chi_{g}^{(3)}(\omega ; \omega,-\omega, \omega)=\frac{\Omega_{2} e^{4} n_{e}}{(\omega+i \gamma)\left(\omega^{2}+\gamma^{2}\right)},
$$

которая обусловлена топологическими свойствами среды.

\section{2. Генерация третьей гармоники}

Новые особенности ГТГ можно рассмотреть, полагая излучение основной волны (накачки) непрерывным. Пусть плоская электромагнитная волна распространяется вдоль оси координат $Z$ в однородной среде. Для 
медленно меняющейся амплитуды волны волновое уравнение сводится к укороченному или редуцированному уравнению [22,23]. Предполагая, что измерением амплитуды накачки можно пренебречь, для медленно меняющейся в пространстве напряженности электрического поля гармоники $\mathbf{E}_{3}(z)$ можно записать следующее уравнение:

$$
\begin{aligned}
-i \frac{\partial}{\partial z} E_{3}= & i a_{0} \mathbf{n} \times \mathbf{E}_{3}+a_{1}\left(\mathbf{E}_{0} \cdot \mathbf{E}_{0}\right) \mathbf{E}_{0} e^{i \Delta k z} \\
& +i a_{2}\left(\mathbf{E}_{0} \cdot \mathbf{E}_{0}\right) \mathbf{n} \times \mathbf{E}_{0} e^{i \Delta k z}
\end{aligned}
$$

где $\Delta k=k(3 \omega)-3 k(\omega)-$ мера нарушения фазового синхронизма и

$$
\begin{aligned}
& a_{0}=\frac{6 \pi \omega \widetilde{\chi}^{(1)}(3 \omega)}{c \sqrt{\varepsilon(3 \omega)}}, \quad a_{1}=\frac{6 \pi \omega \chi^{(3)}(3 \omega)}{c \sqrt{\varepsilon(3 \omega)}}, \\
& a_{2}=\frac{6 \pi \omega \tilde{\chi}^{(3)}(3 \omega)}{c \sqrt{\varepsilon(3 \omega)}}, \quad \tilde{\chi}^{(1)}(3 \omega)=\frac{\Omega_{0} m \omega_{p}^{2}}{3 \omega} .
\end{aligned}
$$

Амплитуды поля накачки считаются постоянными. Пусть ось $Z$ выбрана вдоль единичного вектор $\mathbf{n}$. Если использовать циркулярные компоненты электрического поля накачки $E_{0}^{( \pm)}=E_{0 x} \pm i E_{0 y}$ и гармоники $E_{3}^{( \pm)}=E_{3 x} \pm i E_{3 y}$ (индексами $x$ и $y$ отмечены проекции вектора на оси $X$ и $Y$ ), то уравнение (11) можно записать как систему уравнений

$$
\begin{gathered}
i \frac{\partial}{\partial z} E_{3}^{( \pm)}= \pm a_{0} E_{3}^{( \pm)}-\left(a_{1} \mp a_{2}\right) E_{0}^{2} E_{0}^{( \pm)} e^{i \Delta k z}, \\
E_{0}^{2}=E_{0}^{(+)} E_{0}^{(-)} .
\end{gathered}
$$

Замена переменной $E_{3}^{( \pm)}(z)=A^{( \pm)} \exp \left(\mp i a_{0} z\right)$ приводит к уравнениям

$$
i \frac{\partial}{\partial z} A^{( \pm)}=-\left(a_{1} \mp a_{2}\right) E_{0}^{2} E_{0}^{( \pm)} e^{i \Delta k z} e^{ \pm i a_{0} z} .
$$

Решения получаются (в приближении заданного поля) путем прямого интегрирования при условии, что гармоника в точке $z=0$ отсутствовала. Окончательно

$$
\begin{aligned}
E_{3}^{( \pm)}(z)= & i\left(a_{1} \mp a_{2}\right) E_{0}^{(+)} E_{0}^{(-)} E_{0}^{( \pm)} \frac{2 \sin \left[\left(\Delta k \pm a_{0}\right) z / 2\right]}{\Delta k \pm a_{0}} \\
& \times \exp \left[i\left(\Delta k \mp a_{0}\right) z / 2\right]
\end{aligned}
$$

Важно отметить, что коэффициенты преобразования различно поляризованных по кругу волн оказываются различными. Кроме того, период пространственных осцилляций интенсивности гармоники разный для разных компонент циркулярной поляризации гармоники. Эта асимметрия является следствием предположения о виде вектора кривизны Берри $\boldsymbol{\Omega}(\mathbf{p})=\left(\Omega_{0}+\Omega_{2} \mathbf{p}^{2}\right) \mathbf{n}$. Измерения интенсивностей волн при ГТГ могут дать сведения о параметре $\Omega_{2}$ и подтвердить или опровергнуть рассмотренную здесь модель.

\section{3. Распространение электромагнитной волны в нелинейной среде}

Для эффективной ГТГ и других параметрических взаимодействий требуется выполнение условия фазового синхронизма, в то время как для самовоздействия (автомодуляции, самофокусировки, образования солитонов) такого требования нет. Используя выражения для линейной и нелинейной поляризаций (4) и (10), можно стандартным образом получить укороченное волновое уравнение для медленно меняющейся огибающей волны, распространяющейся в среде, свойства которой описываются рассматриваемой здесь моделью. Считая волну квазигармонической $\mathbf{E}(z, t)=\mathbf{E}_{0}(z, t) e^{-i \omega t+i k z}+\mathbf{E}_{0}^{*}(z, t) e^{-i \omega t-i k z}$, следуя процедуре вывода укороченного волнового уравнения [22,23], можно записать уравнение для огибающей $\mathbf{E}_{0}(z, t)$ в следующем виде:

$$
\begin{aligned}
& i\left(\frac{\partial}{\partial z}+\frac{1}{v_{g}} \frac{\partial}{\partial t}\right) \mathbf{E}_{0}+D \frac{\partial^{2}}{\partial t^{2}} \mathbf{E}_{0}=-\frac{2 \pi \omega}{c \sqrt{\varepsilon(\omega)}} \\
& \times\left\{\chi^{(3)}\left[2\left(\mathbf{E}_{0} \cdot \mathbf{E}_{0}^{*}\right) \mathbf{E}_{0}+\left(\mathbf{E}_{0} \cdot \mathbf{E}_{0}\right) \mathbf{E}_{0}^{*}\right]-i \chi_{g}^{(1)} \mathbf{n} \times \mathbf{E}_{0}-i \chi_{g}^{(3)} \mathbf{n}\right. \\
& \left.\times\left\lfloor 2\left(\mathbf{E}_{0} \cdot \mathbf{E}_{0}^{*}\right) \mathbf{E}_{0}-\left(\mathbf{E}_{0} \cdot \mathbf{E}_{0}\right) \mathbf{E}_{0}^{*}\right\rfloor\right\} .
\end{aligned}
$$

В этом уравнении $v_{g}-$ групповая скорость, $D-$ коэффициент дисперсии групповых скоростей второго порядка $[4,23]$. Для линейного вектора гирации использовано обозначение

$$
\chi_{g}^{(1)} \mathbf{n}=\frac{\Omega_{0} n_{e} e^{2}}{\omega} \mathbf{n} .
$$

При выводе уравнения (12) предполагалось, что $\omega \gg \gamma$, так что все коэффициенты в (12) вещественные. Отказ от этого приближения приведет к учету линейного и нелинейного поглощения.

Если среда не обладает топологическими свойствами (т.е. $\boldsymbol{\Omega}(\mathbf{p})=0$ ), то векторное уравнение (12) для линейно поляризованной или циркулярно поляризованной волны сводится к нелинейному уравнению Шредингера или к системе связанных нелинейных уравнений в случае произвольной поляризации волны. Свойства такой системы уравнений хорошо известны [22-24].

Поведение уединенных волн, описываемых уравнением (12), будет рассмотрено позже. Здесь полезно рассмотреть простейший случай, демонстрирующий роль гиротропии, индуцированной вектором $\Omega(\mathbf{p})$. Пусть ось $Z$ выбрана вдоль единичного вектора и непрерывное излучение плоской поперечной волны распространяется вдоль оси $Z$. Тогда (12) сводится к системе уравнений для циркулярных компонент электрического поля

$$
\begin{aligned}
i \frac{\partial}{\partial z} E_{0}^{( \pm)}= & g_{1} E_{0}^{( \pm)}+\mu\left(\left|E_{0}^{( \pm)}\right|^{2}+\left|E_{0}^{(\mp)}\right|^{2}\right) E_{0}^{( \pm)} \\
& \pm g_{2}\left|E_{0}^{( \pm)}\right|^{2} E_{0}^{( \pm)}
\end{aligned}
$$


где

$$
g_{1}=\frac{2 \pi \omega \chi_{g}^{(1)}}{c \sqrt{\varepsilon(\omega)}}, \quad g_{2}=\frac{2 \pi \omega \chi_{g}^{(3)}}{c \sqrt{\varepsilon(\omega)}}, \quad \mu=\frac{2 \pi \omega \chi^{(1)}}{c \sqrt{\varepsilon(\omega)}} .
$$

Если ввести вещественные амплитуды $a^{( \pm)}$и фазы $\varphi^{( \pm)}$ таким образом, что $E^{( \pm)}=a^{( \pm)} \exp \left[i \varphi^{( \pm)}\right]$, то из (12) можно получить систему для вещественных амплитуд и фаз, причем окажется, что $a^{( \pm)}=a_{0}^{( \pm)}$являются константами, определяемыми из начальных условий. Уравнения для фаз становятся тривиальными, и их решения записываются следующим образом:

$$
\phi^{( \pm)}(z)=\mp\left[g_{1}+g_{2} a_{0}^{( \pm) 2} \pm \mu\left(a_{0}^{( \pm) 2}+2 a_{0}^{(\mp) 2}\right)\right] z+\text { const. }
$$

Следовательно, по мере распространения разность фаз между различно циркулярно поляризованными волнами меняется линейно. Так что, например, $\left|E_{x}\right|^{2}$ изменяется с длиной пройденного пути по гармоническому закону, причем пространственная частота есть

$$
\begin{aligned}
C_{V}= & \left(\varphi^{(-)}-\varphi^{(+)}\right) / z=2 g_{1}+\left(g_{2}-\mu\right) a_{0}^{(+) 2} \\
& +\left(g_{2}+\mu\right) a_{0}^{(-) 2} .
\end{aligned}
$$

Следовательно, величина $C_{V}$ (ее можно назвать нелинейным коэффициентом Верде) асимметрично зависит от интенсивностей полей, отвечающих разным компонентам циркулярной поляризации. В линейном случае асимметрия отсутствует. Полученный результат может быть использован для проверки справедливости рассмотренной модели и для измерения параметров $\Omega(\mathbf{p})$.

\section{Заключение}

Здесь рассмотрена модель нелинейной среды, топологические свойства которой могут быть охарактеризованы вектором кривизны Берри [12-14]. Простые предположения и зависимости от импульса энергии квазичастиц (электронов) и кривизны Берри позволили определить нелинейные восприимчивости третьего порядка. Как примеры рассмотрены ГТГ и нелинейное вращение поляризации волны. Типичной характеристикой таких сред в линейном случае является гиротропия, обусловленная топологическими свойствами динамики электронов и отмеченная в ряде работ, посвященных топологическим изоляторам и полуметаллу Вейля [16-19,25-30]. Это свойство воспроизводится в рассмотренной модели, и предсказывается различие в нелинейных откликах для волн разной циркулярной поляризации.

Справедливость модели определяется условиями справедливости применения квазиклассического приближения, когда движение электронов в кристалле может быть описано движением волнового пакета. В рассмотренной здесь модели учитывалась только одна зона и предполагалось, что межзонными переходами можно пренебречь. От этого приближения можно отказаться, перейдя к обобщенным уравнениям движения волнового пакета (раздел IV в обзорной статье [12]). Здесь также не учитывалось влияние магнитного поля, внешнего поля или поля самой электромагнитной волны. Помимо нелинейных эффектов, связанных с силой Лоренца в уравнениях движения (1), топологические свойства среды проявляются в перенормировке эффективной массы [27-31], что тоже дает вклад в нелинейный отклик среды $[15,32]$. Большое внимание уделяется явлениям переноса в присутствии постоянного или низкочастотного магнитного поля. Распространение таких исследований на случай высокочастотных полей и изучение реакции на импульсные поля, в том числе с малым числом колебаний амплитуды импульса, представляет значительный интерес.

\section{Финансирование работы}

Исследование выполнено при поддержке Российского фонда фундаментальных исследований (грант № 18-02-00921).

\section{Конфликт интересов}

Авторы заявляют, что у них нет конфликта интересов.

\section{Список литературы}

[1] Бломберген Н. Нелинейная оптика. М.: Мир, 1966.

[2] Шен И.Р. Принципы нелинейной оптики. М.: Наука, 1989; Shen Y.R. The Principles of Nonlinear Optics. Wiley-Interscience, 2002. $576 \mathrm{p}$.

[3] Ярив А. Квантовая электроника и нелинейная оптика. М.: Сов. Радио, 1973; Yariv A. Quantum Electronics. New York, London, Sidney: John Wilew \& Song, Inc., 1967.

[4] Кивщарь Ю.С., Агравал Г.П. Оптические солитоны. От волоконных световодов до фотонных кристаллов. М.: ФИЗМАТЛИТ, 2005; Kivshar Yu.S., Agrawal G.P. Optical solitons. From Fibers to Photonic Crystals. Academic Press, 2003.

[5] Ландау Л.Д. // ЖЭТФ. 1937. Т. 7. С. 19.

[6] Гинзбург В.Л., Ландау Л.Д. // ЖЭТФ. 1950. Т. 20. С. 1064.

[7] Абрикосов А.А. Основы теории металлов. М.: ФИЗМАТЛИТ, 2009.

[8] Шмидт В.В. Введение в физику сверхпроводников. М.: МЦНМО, 2000.

[9] Питаевский Л.П. // ЖЭТФ. 1958. Т. 35. С. 408-415.

[10] Gross E.P. // Nuovo Cimento. 1961. V. 20. P. 454.

[11] Киттель Ч. Квантовая теория твердых тел. М.: Наука, 1987; Kittel C. Quantum Theory of Solid. New York, London: John Wilew \& Song, Inc., 1963.

[12] Di Xiao, Ming-Che Chang, Qian Niu. // Rev. Mod. Phys. 2010. V. 82. N 3. P. 1959-2007.

[13] Nagaosa N., Sinova J., Onoda Sh., MacDonald A.H., Ong N.P. // Rev. Mod. Phys. 2010. V. 82. N 2. P. 1539-1592.

[14] Shapere A., Wilczek F. Geometric Phases in Physics. Singapore, Teaneck, N. J.: World Scientific, 1989. 
[15] Маймистов А.И., Ляшко Е.И. // Опт. и спектр. 2019. T. 127. № 5. C. 804-810; Maimistov A.I., Lyashko E.I. // Opt. Spectrosc. 2019. V. 127. N 5. 871-877.

[16] Zebin Qiu, Gaoqing Cao, Xu-Guang Huang. // Phys. Rev. D. 2017. V. 95. P. 036002.

[17] Kotov O.V., Lozovik Yu.E. // Phys. Rev. B. 2018. V. 98. P. 195446.

[18] Zyuzin A.A., Si Wu, Burkov A.A. // Phys. Rev. B. 2012. V. 85. P. 165110

[19] Hofmann J., Das Sarma S. // Phys. Rev. B. 2016. V. 93. P. 241402

[20] Bansil A., Hsin Lin, Tanmoy Das. // Rev. Mod. Phys. 2016. V. 88. P. 021004.

[21] Armitage N.P., Mele E.J., Ashvin Vishwanath. // Rev. Mod. Phys. 2018. V. 90. P. 015001.

[22] Maimistov A.I., Basharov A.M. Nonlinear Optical Waves. Dordrecht, Boston, London: Kluwer Academic Publishers, 1999.

[23] Рыскин Н.М., Трубецков Д.И. Нелинейные волны. М.: УРСС; ЛЕНАНД, 2017.

[24] Ахмедиев Н.Н., Анкевич А. Солитоны. М.: ФИЗМАТЛИТ, 2003; Akhmediev N.N., Ankiewicz A. Solitons. London, Weinheim, NY., Tokyo, Melbourn: Madras Chapman \& Hall, 1997.

[25] Ming-Che Chang, Min-Fong Yang. // Phys. Rev. B. 2009. V. 80. P. 113304.

[26] Murchikova E.M. // J. Phys. A: Math. Theor. 2011. V. 44. P. 045401.

[27] Zyuzin A.A., Burkov A.A. // Phys. Rev. B. 2012. V. 86. P. 115133.

[28] Zyuzin A.A., Zyuzin A.Yu. // Phys. Rev. B. 2017. V. 95. P. 085127.

[29] Huerta L. // J. Phys.: Conference Series. 2016. V. 720. P. 012023.

[30] Barnes E., Heremans J.J., Minic D. // Phys. Rev. Lett. 2016. V. 117. P. 217204.

[31] Son D.T., Spivak B.Z. // Phys. Rev. B. 2013. V. 88. P. 104412.

[32] Imran M., Hershfield S. // Phys. Rev. B. 2018. V. 98. P. 205139. 